\title{
Low Power FSK Receiver Using an Oscillator-Based Injection-Locked Frequency Divider
}

\author{
Rong-Fu Ye, Student Member, IEEE, Tzyy-Sheng Horng, Senior Member, IEEE, and Jian-Ming Wu, Member, IEEE
}

\begin{abstract}
This letter presents a novel frequency-shift keying (FSK) receiver using an oscillator-based injection-locked frequency divider (ILFD), thereby achieving high sensitivity, low dc-offset, and low power consumption. The proposed receiver comprises a low-noise amplifier, a divide-by-2 ring-oscillator-based ILFD, and a subharmonic mixer. Moreover, the proposed receiver is fabricated using $0.18 \mu \mathrm{m}$ CMOS process and consumes $1.1 \mathrm{~mW}$. Measurement results demonstrate that the proposed receiver has a sensitivity of $-83 \mathrm{dBm}$ at $10^{-3}$ bit error rate with $1 \mathrm{Mb} / \mathrm{s}$ data rate in receiving a $2.4 \mathrm{GHz}$ Gaussian FSK signal.
\end{abstract}

Index Terms-CMOS FSK receiver, injection-locked frequency divider (ILFD), ring oscillator, subharmonic mixer.

\section{INTRODUCTION}

I NJECTION-locked oscillators (ILOs) are widely used in frequency-shift keying (FSK) RF receivers [1]-[4]. Given the ability to remove a power-hungry phase-locked-loop (PLL)-based carrier recovery circuitry, the ILO-based FSK receivers are feasible for low power and data rate applications, including wireless sensor networks (WSNs) and wireless body area networks (WBANs). However, despite their potential for low power consumption, these receivers generally incur an insufficient sensitivity due to a limited locking range at low input power levels [2], [3]. In [4], the use of a trifilar transformer splitter in an $L C$-ILO-based FSK receiver can significantly improve sensitivity. However, this design consumes large chip area and power consumption and causes a high dc offset.

This letter presents a novel $2.4 \mathrm{GHz}$ injection-locked-frequency-divider (ILFD)-based FSK receiver, consisting of a low noise amplifier (LNA), a divide-by-2 ring-oscillator-based ILFD, and a subharmonic mixer (SHM). The ILFD can provide larger locking range and lower power consumption than an ILO, thus assisting the proposed receiver to operate with high sensitivity and low power consumption. Moreover, the baseband dc offset is substantially reduced, owing to the use of a SHM in the proposed receiver.

Manuscript received June 21, 2013; revised September 10, 2013; accepted October 15, 2013. Date of publication December 05, 2013; date of current version February 10, 2014. This work was supported by the National Science Council of Taiwan under Grant 100-2622-E-110-001-CC1, Grant 100-2221-E-110-081-MY3, and Grant 100-2221-E-110-082-MY3.

R.-F. Ye and T.-S. Horng are with the Department of Electrical Engineering, National Sun Yat-Sen University, Kaohsiung 80424, Taiwan (e-mail: d963010022@student.nsysu.edu.tw; jason@ee.nsysu.edu.tw).

J.-M. Wu is with the Department of Electronic Engineering, National Kaohsiung Normal University, Kaohsiung 82424, Taiwan (e-mail: jianmingwu @ nknu.edu.tw).

Color versions of one or more of the figures in this letter are available online at http://ieeexplore.ieee.org.

Digital Object Identifier 10.1109/LMWC.2013.2290213

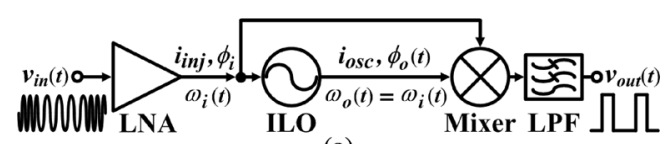

(a)

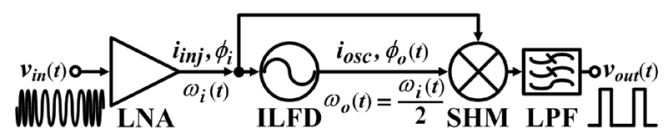

(b)

Fig. 1. Injection-locked FSK receiver architectures. (a) FSK receiver based on an ILO. (b) FSK receiver based on an oscillator-based ILFD.

\section{ILFD-BASED FSK RECEIVER ARCHITECTURE}

Fig. 1(a) shows a conventional ILO-based FSK receiver [3], [4]. Via an injection-locking process, the ILO and mixer perform an FM-to-PM and PM-to-AM conversion, respectively, to accomplish a noncoherent FSK demodulation. However, a dc offset induced by self-mixing in the mixer degrades the baseband signal quality and deteriorates the bit error rate (BER) performance. Moreover, since the ILO is the most power-consuming component in the system, the increasing ILO frequency leads to a larger power consumption of this receiver.

Fig. 1(b) shows the architecture of the proposed ILFD-based FSK receiver, in which $\omega_{i}(t)$ denotes the frequency of a received signal $v_{i n}(t) ; \omega_{o}(t)$ represents the free-running frequency of the oscillator required by the ILFD; $i_{i n j}$ and $i_{o s c}$ stand for the injection and oscillation current, respectively; and $\phi_{i}$ and $\phi_{o}(t)$ refer to the phase of $i_{i n j}$ and $i_{o s c}$, respectively. The comparison with Fig. 1(a) indicates that a divide-by-2 ILFD replaces the ILO in an ILO-based FSK receiver. Accordingly, the ILFD-based FSK receiver has lower power consumption than that of the ILO-based FSK receiver because the former uses an oscillator at half of the frequency of that required by the latter. Furthermore, besides its use in converting $\omega_{o}(t)$ into $2 \omega_{o}(t)$, SHM acts as a phase detector to detect the phase difference between $i_{i n j}$ and $i_{o s c}$. Importantly, since an SHM has a high LO-to-RF isolation, a dc offset caused by self-mixing is markedly decreased. Moreover, the proposed receiver architecture uses a ring-oscillatorbased ILFD that can achieve larger locking range, lower power consumption and less chip area than a conventional $L C$-ILFD.

In the steady state of injection locking, the output voltage $v_{\text {out }}(t)$ of the proposed receiver is given by (1), in which $k_{c}$ denotes the overall gain of the system; $n$ represents the number of stages in the ring oscillator; and $I_{\text {Bias }}$ stands for the biasing current of the ring oscillator [5]. Clearly, (1) indicates an FM-to-AM conversion

$$
v_{\text {out }}(t) \approx \frac{k_{c}}{2}\left[\frac{n \cdot \sin \left(\frac{2 \pi}{n}\right) \cdot I_{\text {Bias }}}{i_{\text {inj }}}\left(\frac{\omega_{o}(t)-\omega_{i}(t) / 2}{\omega_{o}(t)}\right)\right] .
$$




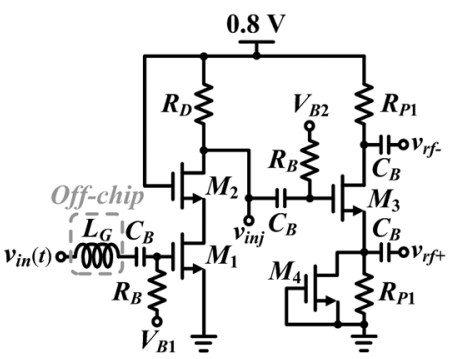

(a)

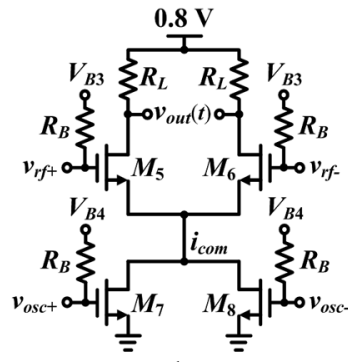

(b)

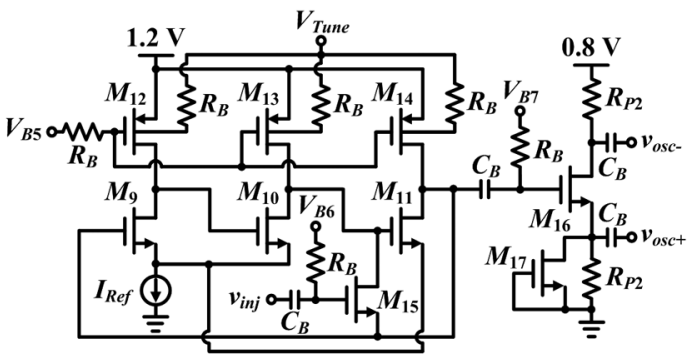

(c)

Fig. 2. Schematics of components in the proposed $2.4 \mathrm{GHz}$ CMOS FSK receiver. (a) LNA with $0.55 \mathrm{~mW}$ power consumption. (b) SHM with $0.21 \mathrm{~mW}$ power consumption. (c) Divide-by-2 ring-oscillator-based ILFD with $0.34 \mathrm{~mW}$ power consumption.

To switch receiving channels, the ring oscillator frequency must be tuned close to the half frequencies of the receiving channels with an offset frequency less than the locking range.

\section{Circuit Design}

In order to further reduce power consumption and supply voltage of the proposed receiver, the sub-threshold and body-biasing techniques [6], [7] are applied to the LNA, active balun, and SHM of this receiver. Fig. 2(a) shows a cascode LNA with an output balun. The cascode LNA comprising transistors $M_{1,2}$ provides an input matching to the $50-\Omega$ source impedance and amplifies the input signal $v_{i n}(t)$, thus enhancing the sensitivity of the receiver. To make a good trade-off among noise figure (NF), gain, and linearity, transistors $M_{1,2}$ of $120-\mu \mathrm{m}$ gate width, a resistor $R_{D}$ of $600 \Omega$, and an external inductor $L_{G}$ of $12 \mathrm{nH}$ are used. Consequently, an NF of $2.5 \mathrm{~dB}$ and a voltage gain of $21 \mathrm{~dB}$ are achieved. The output balun is designed using a resistive loaded common-source amplifier with resistive degeneration. The gate width of transistor $M_{4}$ is chosen as twice the size of that of transistor $M_{3}$ to reduce the load impedance mismatch between the output differential terminals. Moreover, transistor $M_{3}$ with a gate width of $50 \mu \mathrm{m}$, transistor $M_{4}$ with a gate width of $100 \mu \mathrm{m}$, and resistor $R_{P 1}$ of $240 \Omega$ are used in this balun to achieve amplitude and phase imbalance within $0.3 \mathrm{~dB}$ and $1.1^{\circ}$, respectively.

Fig. 2(b) shows a single-balanced SHM, in which the RF stage comprises transistors $M_{5,6}$, and the frequency doubler stage consists of transistors $M_{7,8}$. The common node current $i_{\text {com }}$ at $2 \omega_{o}(t)$ mixes with the RF stage current at $\omega_{i}(t)$. The mixing product current is converted into a differential voltage $v_{\text {out }}(t)$ via resistors $R_{L}$, in which $v_{\text {out }}(t)$ at $2 \omega_{o}(t)$ is filtered out by an external low-pass filter. Transistors $M_{7,8}$ are biased near the threshold voltage to reduce the noise caused by nonideal switching. Moreover, transistors $M_{5,6}$ with a gate width of $40 \mu \mathrm{m}$ and transistors $M_{7,8}$ with a gate width of $35 \mu \mathrm{m}$ are used in this SHM to attain a voltage gain of $15 \mathrm{~dB}$ and an NF of $12 \mathrm{~dB}$.

An attempt is also made to reduce the power consumption of the proposed receiver by using a three-stage design of ring-oscillator-based ILFD [8]. Fig. 2(c) shows an ILFD with an output balun, in which the ILFD consists of transistors $M_{9-11}$ with a gate width of $10 \mu \mathrm{m}$ and transistors $M_{12-14}$ with a gate width of $5 \mu \mathrm{m}$. Since the device size of transistor $M_{15}$ influences the locking range of the ILFD, an optimum gate width of $M_{15}$ is set at $10 \mu \mathrm{m}$. To avoid FM-to-PM distortion, $M_{15}$ is biased near

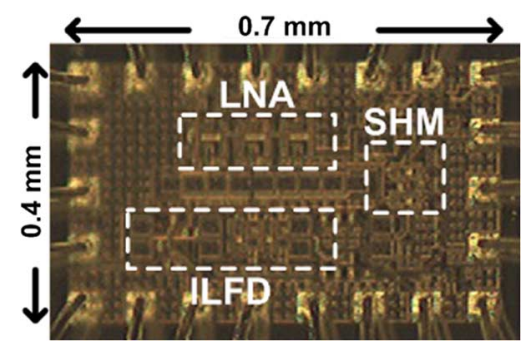

Fig. 3. Chip micrograph of the proposed ILFD-based FSK receiver.

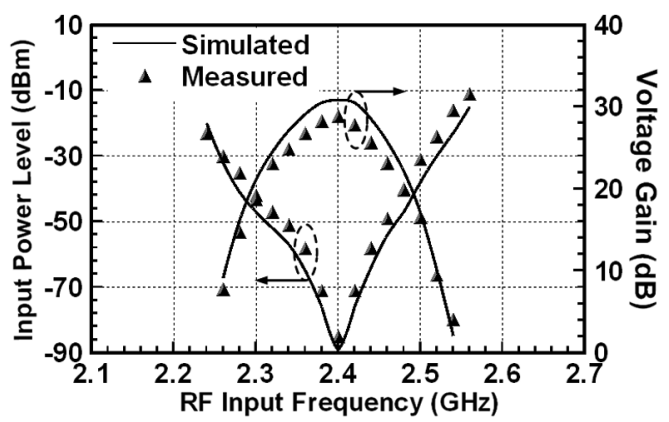

Fig. 4. Comparison of locking range and voltage gain between simulation and measurement for the proposed ILFD-based FSK receiver.

the weak inversion region to increase conversion loss while receiving an excessively high input voltage $v_{i n j}(t)$. Moreover, to ensure that ILFD is stably locked in the tuning bandwidth by a received signal, the locking range of the ILFD is designed much larger than the frequency shift caused by the process, voltage and temperature (PVT) variations. Furthermore, a biasing circuit with temperature compensation is applied to ILFD for reducing frequency drift due to temperature variation. Notably, varying the body voltage $V_{\text {Tune }}$ and biasing current $I_{\text {Ref }}$ allows a maximum tuning range of 2.2 to $2.6 \mathrm{GHz}$ for the ILFD. In the output balun, transistor $M_{16}$ with a gate width of $40 \mu \mathrm{m}$, transistor $M_{17}$ with a gate width of $80 \mu \mathrm{m}$, and resistor $R_{P 2}$ of $220 \Omega$ are used to achieve an amplitude mismatch and phase error of less than $0.4 \mathrm{~dB}$ and $1.3^{\circ}$, respectively.

\section{EXPERIMENTAL RESULTS}

The proposed $2.4 \mathrm{GHz}$ ILFD-based FSK receiver was fabricated using $0.18 \mu \mathrm{m}$ CMOS technology. Fig. 3 shows a micrograph of the implemented chip, which occupies an area of $0.28 \mathrm{~mm}^{2}$ including pads. A test buffer for ILFD is also integrated in this chip. An external operational amplifier was used during measurements, and the power dissipation $P_{\text {diss }}$ of the chip alone is $1.1 \mathrm{~mW}$. The measured input matching values of 


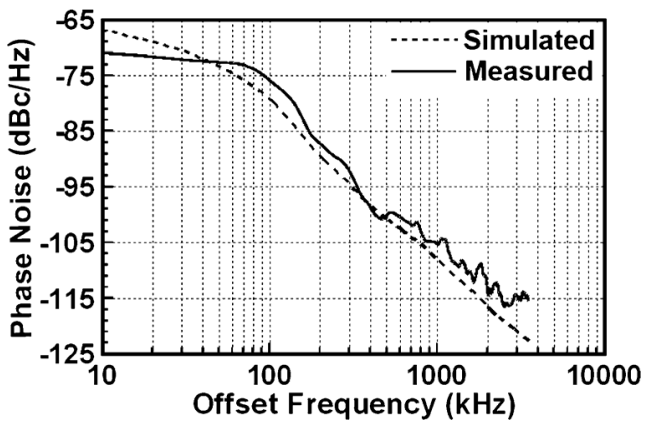

Fig. 5. Simulated and measured phase noise of the unlocked ILFD at $1.2 \mathrm{GHz}$.

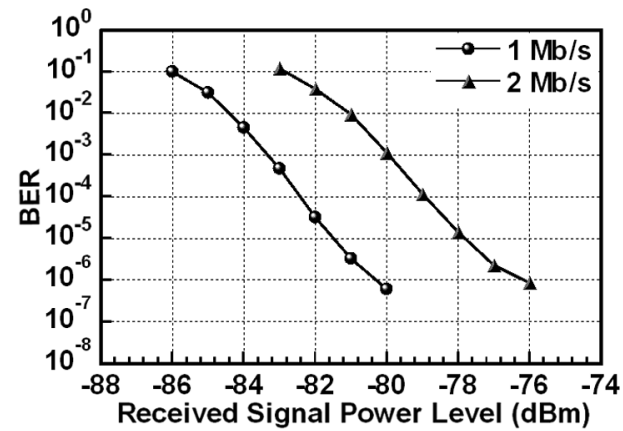

Fig. 6. Measured BER of the GFSK signals with different data rates.

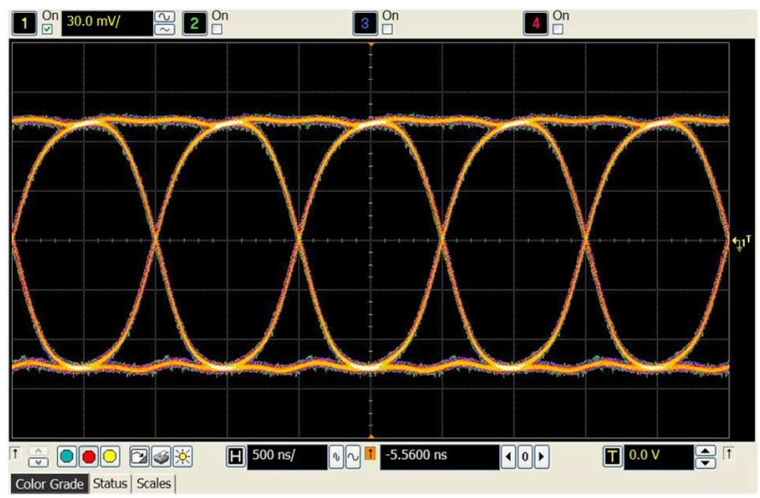

Fig. 7. Measured eye diagram of the demodulated GFSK signal at $1 \mathrm{Mb} / \mathrm{s}$.

TABLE I

Comparison With Previous 2.4 GHz GFSK Receiver Designs

\begin{tabular}{|c|c|c|c|c|}
\hline Reference & $\begin{array}{c}\text { Sensitivity }(\mathrm{dBm}) @ \\
10^{-3} \mathrm{BER} \& 1 \mathrm{Mbps}\end{array}$ & $\begin{array}{c}\text { Area } \\
\left(\mathbf{m m}^{2}\right)\end{array}$ & $\begin{array}{c}\text { P }_{\text {diss }} \\
(\mathrm{mW})\end{array}$ & $\begin{array}{c}\text { CMOS } \\
(\mathbf{n m})\end{array}$ \\
\hline This work & $-83 \mathrm{dBm}$ & 0.28 & $1.1^{\mathrm{a}}$ & 180 \\
\hline$[4]$ & $-81 \mathrm{dBm}$ & 0.43 & $1.8^{\mathrm{a}}$ & 90 \\
\hline$[9]$ & $-92 \mathrm{dBm}$ & 3 & $27.9^{\mathrm{b}}$ & 130 \\
\hline$[10]$ & $-82.5 \mathrm{dBm}$ & N/A & $9.12^{\mathrm{c}}$ & 180 \\
\hline
\end{tabular}

${ }^{a}$ exclude external operation amplifier

${ }^{b}$ include variable gain LNA, demodulator, synthesizer, IF amplifier, ADC, and power management circuits

${ }^{c}$ include variable gain LNA, demodulator, synthesizer, and IF amplifier

the receiver are below $-13 \mathrm{~dB}$ over $2.3-2.5 \mathrm{GHz}$. As a continuous-wave (CW) measurement result, Fig. 4 shows the input power level and voltage gain of the receiver operating in the boundary of the locking range. Measurement results indicate that the proposed receiver has a minimum lockable signal level of $-89 \mathrm{dBm}$ at a voltage gain of $29 \mathrm{~dB}$. Fig. 5 shows the phase noise of the unlocked ILFD at $1.2 \mathrm{GHz}$, in which the measured phase noise at $1 \mathrm{MHz}$ offset is $-105 \mathrm{dBc} / \mathrm{Hz}$.

The proposed receiver exhibits an input $1 \mathrm{~dB}$ compression point of $-25 \mathrm{dBm}$. Its sensitivity is evaluated using a Gaussian FSK (GFSK) signal with data rates of 1 and $2 \mathrm{Mb} / \mathrm{s}$. Fig. 6 plots the measured BER performance at these data rates. The sensitivity for a BER of $10^{-3}$ at a data rate of 1 and $2 \mathrm{Mb} / \mathrm{s}$ is -83 and $-80 \mathrm{dBm}$, respectively. Fig. 7 displays a clear eye diagram with a low peak-to-peak jitter while the receiver demodulates a received $2.4 \mathrm{GHz}$ GFSK signal at a data rate of $1 \mathrm{Mb} / \mathrm{s}$ and an input power of $-70 \mathrm{dBm}$. Table I compares the sensitivity and power consumption performance of this work with that of previous ILO- or PLL-based designs for 2.4 GHz GFSK receivers [4], [9], [10]. Obviously, the proposed receiver outperforms the others in reducing power consumption while providing comparable sensitivity.

\section{CONCLUSION}

In this letter, a $2.4 \mathrm{GHz}$ CMOS FSK receiver based on a divide-by-2 ILFD and a single-balanced SHM is presented. The ILFD uses a ring oscillator to achieve large locking range and low power consumption. Moreover, the SHM provides a high LO-to-RF isolation to prevent the dc offset. The presented receiver can therefore be highly sensitive and energy-efficient. Measurement results exhibit a sensitivity of $-83 \mathrm{dBm}$ and a dissipated power of $1.1 \mathrm{~mW}$ when the receiver is used to detect a GFSK signal with a data rate of $1 \mathrm{Mb} / \mathrm{s}$.

\section{REFERENCES}

[1] E. Main and D. Coffing, "An FSK demodulator for Bluetooth applications having no external components," IEEE Trans. Circuits Syst. II: Express Briefs, vol. 49, no. 6, pp. 373-378, Jun. 2002.

[2] C.-S. Wang, K.-D. Chu, and C.-K. Wang, "A $0.13 \mu \mathrm{m}$ CMOS 2.5 Gb/s FSK demodulator using injection-locked technique," in Proc. IEEE Radio Freq. Integr. Circuits Symp., Jun. 2009, pp. 563-566.

[3] K. Kawasaki, Y. Akiyama, K. Komori, M. Uno, H. Takeuchi, T. Itagaki, Y. Hino, Y. Kawasaki, K. Ito, and A. Hajimiri, "A millimeterwave intra-connect solution,” IEEE J. Solid-State Circuits, vol. 45, no. 12, pp. 2655-2666, Dec. 2010.

[4] R.-F. Ye, T.-S. Horng, and J.-M. Wu, "Ultralow power injection-locked GFSK receiver for short-range wireless system," IEEE Trans. Circuits Syst. II: Express Briefs, vol. 52, no. 11, pp. 706-710, Nov. 2012.

[5] R. J. Betancourt-Zamora, S. Verma, and T. H. Lee, "1 GHz and 2.8 GHz CMOS injection-locked ring oscillator prescalers," in Proc. IEEE. VLSI Circuits Symp., Jun. 2001, pp. 47-50.

[6] A. V. Do, C. C. Boon, M. A. Do, K. S. Yeo, and A. Cabuk, "A weakinversion low-power active mixer for $2.4 \mathrm{GHz}$ ISM band applications," IEEE Microw. Wireless Compon. Lett., vol. 19, no. 11, pp. 719-721, Nov. 2009.

[7] C.-P. Chang, J.-H. Chen, and Y.-H. Wang, "A fully integrated $5 \mathrm{GHz}$ low-voltage LNA using forward body bias technique," IEEE Microw. Wireless Compon. Lett., vol. 19, no. 3, pp. 176-178, Mar. 2009.

[8] K. Yamamoto and M. Fujishima, "A 44- $\mu \mathrm{W} 4.3 \mathrm{GHz}$ injection-Locked frequency divider with $2.3 \mathrm{GHz}$ locking range," IEEE J. Solid-State Circuits., vol. 40, no. 3, pp. 671-677, Mar. 2005.

[9] D. Weber, W. W. Si, S. Abdollahi-Alibeik, M. Lee, R. Chang, H. Dogan, S. Luschas, and P. Husted, "A single-chip CMOS radio SoC for v2.1 Bluetooth applications," in IEEE Int. Solid-State Circuits Conf. Dig., Feb. 2008, pp. 364-365.

[10] P. A. Dal Fabbro, T. Pittorino, C. Kuratli, R. Kvacek, M. Kucera, F. Giroud, S. Tanner, F. Chastellain, A. Casagrande, A. Descombes, V. Peiris, P.-A. Farine, and M. Kayal, “A 0.8 V 2.4 GHz $1 \mathrm{Mb} / \mathrm{s}$ GFSK RF transceiver with on-chip dc-dc converter in a standard $0.18 \mu \mathrm{m}$ CMOS technology," in Proc. IEEE Eur. Solid-State Circuits Conf., Sep. 2010, pp. $458-461$. 